\title{
Aproximación Al Discurso de la Acogida y la Solidaridad en Torno al Rescate Del Buque Aquarius en Una Muestra DE LA PRENSA EsPaÑola
}

\section{Approaching the discourse of bospitality and solidarity regarding the rescue of the ship Aquarius in a sample of the spanish press}

\section{Dra. María MARTÍNEZ LIROLA}

Profesora Titular de la Universidad de Alicante y Research Fellow de la University of South Africa (UNISA), España/Sudáfrica

E-mail: maria.lirola@ua.es

(iD) https://orcid.org/0000-0002-6427-425X

Fecha de recepción del artículo: 19/03/2020

Fecha de aceptación definitiva: 21/10/2020

\section{RESUMEN}

Este estudio analizará las principales características lingüísticas de una muestra de noticias con el fin de profundizar en los discursos que presenta la prensa española en torno a la ayuda humanitaria que ofreció España para acoger al buque Aquarius en 2018. Se ofrece un análisis crítico del discurso de acogida y solidaridad que se genera en la prensa española en torno al rescate del Aquarius y se profundiza en el binomio "nosotros-ellos".

El corpus consiste en todas las noticias publicadas en la versión electrónica de los periódicos españoles El País, ABC, El Mundo y La Vanguardia desde el 10 de junio al 10 de julio de 2018. Los resultados revelan que se crea un discurso relacionado con la acogida y la solidaridad de los/as inmigrantes pero que este lleva consigo potenciar el binomio "nosotros-ellos" en el que nosotros, España, se presenta de un modo muy positivo, en contraste con Italia.

Palabras clave: Inmigrantes; prensa; análisis crítico del discurso; buque Aquarius; discurso de la solidaridad.

\begin{abstract}
This study will analyse the main linguistic characteristics of a news sample in order to deepen on the discourses that the Spanish press presents regarding the humanitarian aid that Spain offered to host the Aquarius ship in 2018. This article offers a critical analysis of the discourse of hospitality and solidarity that is generated in the Spanish press regarding the rescue of the Aquarius ship and it deepens on the "we-they" binomial.
\end{abstract}


The corpus consists of all the news published in the electronic version of the Spanish newspapers, El País, ABC, El Mundo and La Vanguardia from June 10 to July 10, 2018. The results reveal that a discourse related to the reception and the solidarity of the immigrants is created, but that this brings with it the strengthening of the binomial "we-they" in which we, Spain, present ourselves in a very positive way, in contrast with Italy.

Keywords: Immigrants; press; critical discourse analysis; Aquarius ship; the discourse of solidarity.

\section{Introducción}

Los medios de comunicación tienen un papel fundamental en la creación de opinión pública al contribuir de un modo directo a configurar las opiniones y las percepciones de las personas sobre temas políticos y sociales, entre los que se encuentra la inmigración (Bednarek y Caple, 2017; Milner, 2012; Nelson y Davis-Wiley, 2018; Romano, 2019). En este sentido, la prensa contribuye a moldear nuestro pensamiento y al cambio sociopolítico al promover tanto la crítica constructiva como la reflexión sobre las realidades sociales que transmiten las noticias, como es la llegada de inmigrantes a las costas españolas.

El modo en que la prensa narra la llegada de inmigrantes y construye su representación tiene un efecto directo en cómo se percibe la inmigración como fenómeno social y en cómo se construye discursivamente la llegada de inmigrantes de forma irregular para los/as lectores/as. Los inmigrantes suelen representarse como héroes, como víctimas o como amenaza fundamentalmente (Martínez Lirola, 2016). Esto tiene una repercusión tanto en la percepción del grupo mayoritario, la sociedad española, como en las actitudes sociales y en los valores que se generan en torno a la inmigración.

La negativa del gobierno italiano en junio de 2018 a que el buque Aquarius atraca en costas italianas con 629 personas a bordo generó una crisis en los países del Mediterráneo. En esta situación, el recién nombrado presidente de España Pedro Sánchez y su gobierno ofrecieron un puerto español para su desembarque al considerarse esta una situación de emergencia humanitaria. El rescate tuvo lugar cuando estas 629 personas viajaban a costas europeas en condiciones infrahumanas. Los medios de comunicación en general y la prensa en particular tuvieron un papel fundamental en la transmisión de la decisión del gobierno de España de acoger a los inmigrantes a bordo del buque Aquarius en el puerto de Valencia, tanto en la narración de la travesía, como del rescate y la posterior llegada a España.

Este artículo pretende ofrecer una aproximación al discurso que utiliza la prensa para narrar el rescate del buque Aquarius, profundizar en el discurso en torno a la acogida y la solidaridad de los/as inmigrantes y analizar cuáles son sus características. Este tipo de discurso no suele ser habitual en la prensa española pues el discurso que predomina es el de rechazo a la inmigración al emplear estrategias discursivas que potencian la victimización y la criminalización (Bañón, 2002; Casero, 2007; Martínez Lirola, 2016), de modo que no se fomenta la integración de las personas inmigrantes.

La terminología que las personas utilizan para referirse a otras es sin duda una herramienta para humanizar o deshumanizar a los demás. La investigación actual muestra que la forma que se emplea para referirse a los inmigrantes contribuye a su discriminación y a fomentar el racismo (Caicedo, 2016; Nelson y Davis-Wiley, 2018). La lengua que se emplea para hacer referencia a los inmigrantes puede considerarse 
ejemplo de "valoración" (appraisement) (van Leeuwen, 2008), al tratarse claramente de una lengua que evalúa, siguiendo a van Leeuwen, (2008, p. 45) 'social actors are appraised when they are referred to in terms which evaluate them as good or bad, loved or hated, admired or pitied'. En este sentido, este estudio contribuye a ofrecer una aproximación al discurso de la acogida y la solidaridad que surge en la prensa española con el rescate del buque Aquarius. Se prestará atención al léxico empleado para hacer referencia a los inmigrantes rescatados.

La hipótesis de partida de este artículo es la siguiente: se observa un discurso en la prensa española relacionado con la necesidad de acoger y ser solidarios/as con ante la llegada del buque Aquarius a Valencia en 2018. La pregunta de investigación que se trata de responder en este artículo es: ¿Surge en la prensa española un discurso de la acogida y la solidaridad en torno al rescate del buque Aquarius? Los objetivos de la investigación son: 1. Analizar las noticias relacionadas con la travesía y el rescate del buque Aquarius en una muestra de la prensa española con el fin de observar las características principales del discurso que se emplea. 2. Ilustrar el discurso de la acogida y la solidaridad que surge en torno a esta crisis humanitaria con ejemplos de las noticias analizadas.

Consideramos fundamental visibilizar la realidad social de la inmigración debido a que se trata de un tema global de vital importancia para los países de acogida de la Unión Europea, especialmente tras la crisis de refugiados que surge en el año 2016 cuando más de un millón de migrantes irregulares llegaron a costas del mediterráneo sólo por mar, y alrededor de 34.000 por vía terrestre (ACNUR, 2016). En el informe de 2017 (ACNUR, 2017) se señala que hay 25,4 millones de refugiados, 2,9 millones más que en 2016. También ha de vincularse a los Objetivos del Desarrollo Sostenible (ODS) que Naciones Unidas (2015) propone para el año 2030. Concurrimos con Hagen-Zanker, Mosler Vidal y Sturge (2017) en que dichos objetivos han de contribuir a que las sociedades sean más inclusivas y justas, tal y como señala el objetivo 16: 'Promover sociedades pacíficas e inclusivas para el desarrollo sostenible, facilitar acceso a la justicia para todos y crear instituciones eficaces, responsables e inclusivas a todos los niveles'.

La inmigración es un tema social actual muy complejo debido a que los inmigrantes dejan sus países de origen y exponen sus vidas en busca de un futuro mejor debido a las difíciles situaciones sociales que viven en sus países de origen, caracterizada fundamentalmente por la pobreza o la guerra (Martínez Lirola, 2017a). La decisión del gobierno de España del 10 de junio de 2018 de acoger a los inmigrantes del Aquarius ante la negativa de Italia es un hecho determinante para el fomento de la acogida y la solidaridad, tal y como se señalará en este artículo.

\section{Estado de la cuestión}

El número de personas inmigrantes que han llegado a España en las últimas décadas ha sido elevado debido a las guerras, la pobreza y las violaciones de derechos humanos (DDHH) en sus países de origen (Arango, 2002); el caso de Siria sería uno de los ejemplos más recientes. La mayoría de los inmigrantes proceden de Siria, Irak, Libia, Afganistán, Pakistán, Myanmar e Irán. También hay muchos albanos, kosovares, eritreos, sudaneses, somalíes, nigerianos y otras personas proceden de Níger, Chad y Camerún, entre otros (Pries, 2018). 
Son múltiples los estudios tanto nacionales como internacionales que se han llevado a cabo en los últimos años relacionados con la representación de los inmigrantes en los medios de comunicación (Bañón Hernández, 2007; Bañón 2019; Bañón y Romero, 2013; Cruz Moya, 2005, 2020; KhosraviNik, 2009, 2010; Martínez Lirola, 2016, 2017a, 2017b; Moore, Gross y Threadgold, 2012; van Dijk, 2005a, 2005b; Wodak y Reisigl, 2001).

Estos estudios coinciden en que la tendencia general es a representar a los inmigrantes de forma negativa, al presentarlos como problema social (Konstantinidou y Michailidou, 2014) o como personas deshumanizadas (Solves Almela y Arcos Urrutia, 2020), aspecto que contribuye a generar actitudes de rechazo en la sociedad de acogida. El hecho de que la lengua que se emplea para hacer referencia a las personas inmigrantes tienda a ser negativa (Bañón Hernández, 2007; Martínez Lirola, 2017a) no favorece la integración, e incluso a contribuye a potenciar un discurso del odio (Niñoles Galvañ y Ortega-Giménez, 2020). Además, se destaca que los inmigrantes llegan en situación ilegal y por lo tanto carecen del estatus de ciudadanía (Anderson, 2013).

Hay una relación directa entre el discurso que se genera en torno a una realidad social determinada, la inmigración, y el contexto que lo enmarca. Es decir, discurso y contexto se relacionan directamente pues, si el contexto fuera distinto, el discurso también cambiaría. Esta idea es relevante para este artículo porque la llegada de los inmigrantes en el buque Aquarius al puerto de Valencia genera discursos distintos a los tradicionales que asocia inmigración con problemas y con racismo. En este caso, al considerarse una emergencia humanitaria, surgen discursos relacionados con la acogida y la solidaridad (vid. apartado 4).

Consideramos fundamental hacer referencia a los conceptos de acogida y solidaridad por tratarse de dos realidades importantes para el análisis sociológico y discursivo actual. Estos dos conceptos han sido analizados y tratados en recientes trabajos sobre discurso, inmigración y políticas migratorias relacionados con la crisis de refugiados e inmigrantes en los últimos cinco años en Europa (Agustín García y Bak Jørgensen 2016, 2018). La solidaridad lleva consigo nuevas configuraciones de las relaciones sociales y políticas pues con ella se pretende acoger a las personas refugiadas y detectar mecanismos de exclusión social y cultural, en un panorama poco alentador, tal y como señalan Agustín García y Bak Jørgensen, (2016, p. 223):

«From a nation state perspective the current situation in Europe does not inspire optimism. [...] European migration policies are not faring much better. They are being tightened on all dimensions: control of undocumented immigrants and refugees, quotas for asylum seekers, and discrimination against second-and even third-generation immigrants».

Se puede hablar de una crisis de las personas refugiadas en el Mediterráneo debido a los flujos masivos que se han observado en los últimos años (Arenas-Hidalgo, 2016). Más de un millón de refugiados e inmigrantes han llegado a Europa en los últimos cinco años por lo que nos encontramos ante el mayor desplazamiento de personas desde la Segunda Guerra Mundial (Aguilar-Idáñez y Buraschi, 2017).

En general los trabajos señalan que las referencias a los refugiados no tienen en cuenta los imperativos humanitarios que conceden importancia a la salvación de vidas desde que salen de sus países, así como la asistencia y protección una vez que llegan a los países de acogida (Amnistía Internacional, 2015; Pries, 2018). Esto indica que desde la crisis de refugiados que surge en el año 2015 se muestra que la Unión Europea tiene unas políticas de asilo y migración incompletas (Faure, Gavas y Knoll, 
2015), pues sus principales intereses son económicos y centrados en la causa de la crisis que lleva a las personas a desplazarse.

Desde dicha crisis surge un discurso de solidaridad europea que se fundamenta en las razones humanitarias que justifican la ayuda y la acogida que han de recibir las personas refugiadas. Esto potencia un discurso relacionado con la construcción de una ciudadanía cívica para la que la empatía y la tolerancia son importantes siempre que sean 'ellos', las personas que llegan de otros países, las que se adapten (Reina-Vanegas, 2016). El discurso solidario se justifica por el hecho de que los refugiados necesitan protección, en palabras de Osorio Ramírez (2012, p. 96):

"Los refugiados son personas desterritorializadas, productos de un sistema social de anomia total y para los cuales las organizaciones transnacionales y nacionales definen el estatus y los criterios de inclusión o exclusión. Los refugiados necesitan protección porque sus derechos han sido violados; víctimas de la guerra, han perdido todo tipo de vínculos: social, político y familiar entre otros».

Además, la solidaridad se manifiesta de distintas formas y por medio de diferentes prácticas que llevan consigo planteamientos para el desarrollo de sociedades más inclusivas en las que los espacios y las relaciones se configuran de maneras diferentes, tal y como señalan Agustín García y Bak Jørgensen (2018, p. 30): "Solidarities produce new ways of configuring political relations and spaces".

\section{Marco teórico, datos y metodología}

Con el fin de analizar los discursos de acogida y solidaridad que surgen en torno al rescate del buque Aquarius, utilizaremos la perspectiva que nos ofrece el análisis crítico del discurso (ACD), al profundizar en las relaciones entre el discurso y el contexto de fenómenos sociales complejos como puede ser la inmigración. El ACD permite deconstruir las ideologías y el poder que surgen en las situaciones sociales con el fin de desenmascarar situaciones sociales injustas en las que las personas son oprimidas (KhosraviNik, 2014; Wodak, 2011; Wodak y Meyer, 2009).

La lengua manifiesta el poder de las personas o instituciones a través del discurso, debido a la relación estrecha que existe entre la lengua y las cuestiones sociales, en palabras de Wodak (2011, p. 52): 'The constant unity of language and other social matters ensures that language is entwined in social power in a number of ways: language indexes power and expresses power; language is involved where there is contention over and a challenge to power'. En este sentido, discurso, poder, hegemonía e ideología han de considerarse aspectos clave en la perspectiva ecléctica que es el ACD. Así, se potencia en todo momento una perspectiva crítica del objeto de estudio (Fairclough, 1995), que en este estudio es la realidad migratoria.

Se han seleccionado las ediciones digitales de tres principales periódicos nacionales españoles para preparar el corpus de ejemplos: El País, considerado un periódico de izquierdas, $A B C$ se enmarca dentro de la derecha y El Mundo es considerado liberal. ${ }^{1}$ El hecho de que la alcaldesa de Barcelona sea Ada Colau, persona que ofreció

1. Conviene precisar que estas ideologías surgen a partir de la autodefinición que hacen los periódicos sobre sus orientaciones ideológicas. Sin embargo, las posiciones ideológicas de los medios se han ido transformando bajo los diferentes gobiernos y algunos de ellos han tomado posiciones más críticas frente al gobierno de Sánchez (en comparación con el gobierno Zapatero que también 
Barcelona como ciudad refugio, nos pareció interesante para incluir la edición digital del periódico catalán La Vanguardia en nuestro corpus por la posible relación entre esta ideología relacionada con la acogida y la representación de los inmigrantes rescatados.

El género periodístico al que pertenecen los textos analizados es la noticia. El número total de noticias recopiladas es de 176; distribuidas del modo que se puede ver en la tabla 1. Consideramos que se trata de un número adecuado de textos para ofrecer ejemplos y obtener algunas conclusiones. Hemos de señalar que la recopilación de las noticias comprende desde el 10 de junio al 10 de julio de 2018. Este período de tiempo comienza con el ofrecimiento de España de ofrecer Valencia como puerto seguro, la posterior llegada de los inmigrantes y las medidas llevadas a cabo para acogerlos en distintos lugares del país tras su llegada a Valencia.

Tabla 1. Distribución del corpus.

\begin{tabular}{|l|l|l|}
\hline Periódico & $\begin{array}{l}\text { Número de noticias } \\
\text { recopiladas }\end{array}$ & Porcentaje del corpus \\
\hline ABC & 55 & 31,25 \\
\hline El Mundo & 29 & 16,47 \\
\hline El País & 43 & 24,43 \\
\hline La Vanguardia & 49 & 27,84 \\
\hline Noticias totales & 176 & $100 \%$ \\
\hline
\end{tabular}

Fuente: Elaboración propia.

La aproximación que ofrecemos es principalmente cualitativa, siguiendo con la tradición del ACD. Sin embargo, también se hace referencia a algunos datos cuantitativos de modo que ambos análisis se complementan, de ahí que en la tabla anterior se hayan presentado algunos datos cuantitativos relacionados con la estructura del corpus. Además, en la recopilación del corpus, se prestó atención a las distintas voces que aparecían en las noticias. En este sentido optamos por el debate social como macrogénero (Bañón, 2002) para analizar la multiplicidad de voces y de actitudes que la prensa refleja a través de ellas. Esto es fundamental pues la cobertura mediática de la realidad migratoria y de los temas relacionados con este debate social influye en cómo la sociedad percibe a las personas migrantes.

Al recopilar los distintos testimonios se observó que apenas había ejemplos de discurso pronunciado por inmigrantes $(3,22 \%)$ frente a un gran número de mensajes pronunciados por personas con cargos políticos ( $50 \%$ del total de los testimonios) y, en menor número, por personas pertenecientes a Organizaciones No Gubernamentales (ONGs) (46,77\%). El hecho de que estas voces hablan desde una perspectiva particular afecta al análisis. En este sentido, no es casualidad que los representantes de ONGs utilizan metáforas que comportan un discurso de solidaridad, por ejemplo.

era del PSOE, por ejemplo). En este sentido, El País ha sido cuestionado recientemente por no representar más los intereses de la llamada 'izquierda'. El Mundo, tampoco es percibido actualmente como 'liberal' por lo que no se observan grandes diferencias ideológicas entre los diferentes periódicos, como se podrá ver en los ejemplos que se presentan en el análisis. 
$\mathrm{Al}$ hacer referencia a las voces, hemos de señalar que en el corpus se presta atención a los distintos actores sociales que aparecen, es decir, a los inmigrantes, las personas con cargos políticos y las pertenecientes a ONGs.

\section{Análisis del discurso de la acogida y la solidaridad de españa en contraste con italia: deconstrucción del binomio "nosotros-ellos" (españa-italia)}

Antes de centrarnos en el discurso que elogió la decisión de España de acoger a las personas a bordo del Aquarius por tratarse de un discurso novedoso en la prensa española, vamos a referirnos brevemente al léxico empleado para hacer referencia a los inmigrantes durante el viaje de rescate.

Al analizar la forma en que la prensa se refiere a las personas que van a bordo del buque Aquarius y las naves Orione y Dattilo, se observa que predomina el uso de la palabra inmigrantes, en 629 ocasiones, seguida de personas en 355 ejemplos y refugiados en 335 casos. Además, también hay ejemplos en los que se les nombra como los rescatados (164²), los migrantes (141), los náufragos (27), los desplazados (5), los acogidos (14) los migrantes rescatados (14), las personas rescatadas (9), los 630 inmigrantes rescatados (2) y los pasajeros del barco (2). En los dos ejemplos que siguen, los adjetivos empleados contribuyen sin duda a presentar a los inmigrantes en una situación vulnerable y por lo tanto potencian actitudes solidarias al presentarlos como víctimas: "las desesperadas, malnutridas y dañadas personas" (La Vanguardia, 14-06-2018) y "los desventurados migrantes" (ABC, 13-06-2018).

Aunque en las noticias analizadas predomina un discurso de acogida y no se encuentran referencias a la situación ilegal o irregular de los inmigrantes que llegan, hay noticias en las que sí se hace referencia a la inmigración como problema, en concreto en nueve casos, es decir en el 5,11\% de las noticias analizadas, aspecto que indica que no se trata de un rasgo informativo relevante: "problema de los flujos migratorios" (El Mundo, 14-06-2018) o "la emergencia migratoria" ( $A B C, 13-06-2018)$.

Es muy habitual que se haga referencia a cifras indicando el número de inmigrantes que llegan en las noticias sobre este tema. En este caso, cada noticia hace referencia a la llegada a Valencia de 629 personas y un bebé que nace a bordo, es decir, un total de 630 seres humanos. No hemos de acostumbrarnos a las cifras porque detrás de ellas hay personas e historias de vida, que en muchas ocasiones son muy duras. Otra cifra recurrente en las noticias analizadas es la que hace referencia a los menores, un total de 88 referencias; se precisa el número y el hecho de que no están acompañados, como podemos observar en el siguiente ejemplo: "Los 123 menores "no acompañados" serán llevados a centros o familias de acogida. (ABC, 13-06-2018).

Las características lingüísticas presentadas en los párrafos anteriores contrastan con las estrategias de referencia a la hora de la llegada a España. Una vez que los inmigrantes llegan a Valencia, se observan ejemplos de un discurso que sugiere la idea de invasión pues encontramos referencias a "la llegada masiva" de inmigrantes como en El Mundo (18-06-2018), en concreto en tres noticias del corpus, lo que supone un 1,70\%, y al "efecto llamada", en quince ocasiones, es decir, afecta al 8,50\% de las

2. Entre paréntesis se ofrece en esta palabra y en las que siguen el número total de ocasiones en las que cada palabra aparece en el corpus de noticias analizadas. 
noticias analizadas, como en $A B C$ (18-06-2018). La referencia a la inmigración de forma negativa es evidente en ejemplos en los que se habla del drama migratorio o de la crisis migratoria mencionados en veinticuatro ocasiones, como por ejemplo en La Vanguardia (18-06-2018).

Estas formas de referirse a la inmigración en este contexto son relevantes porque alertan a la sociedad de acogida sobre el hecho de que son muchos los inmigrantes que llegan, hecho que implica recursos, posibles problemas de convivencia, etc. Cuando llegan los barcos, encontramos referencias a los inmigrantes de una manera neutra al usar el calificativo de "Los recién llegados", colocación que se emplea en veintiséis ocasiones, por ejemplo, en El País (18-06-2018).

Tras estos párrafos introductorios, vamos a analizar el discurso de acogida y solidaridad de España que surge en la prensa española. La negativa del gobierno italiano a abrir sus puertos a la llegada del Aquarius y el ofrecimiento de España al desembarco en Valencia, potencia una imagen positiva y solidaria de España en general y de Valencia en particular. Nos encontramos, por tanto, ante un nuevo binomio "nosotros-ellos": en este caso "los otros" no son los inmigrantes, como suele ser habitual en las noticias relacionadas con la inmigración en la prensa española (van Dijk, 2005a), sino que es otro país europeo, Italia, el que se presenta como distinto a nosotros, mucho menos solidario y acogedor. Así, "nosotros" es España, que acoge y es solidaria, y "ellos" es el gobierno italiano, que rechaza ofrecer ayuda humanitaria en una situación de emergencia. En este sentido, nuestro análisis se centra en observar cuál es el sujeto solidario, es decir, España y en cómo se construye la solidaridad y cómo se argumenta y contraargumenta en el caso de Italia. Los primeros ejemplos muestran la construcción de la solidaridad y la acogida dónde el sujeto es España y qué estrategias lingüísticas se usan para ello.

Cada elección contribuye a crear relaciones entre la lengua y la sociedad, que en esta ocasión se focaliza en ofrecer una imagen positiva de la sociedad española (Richardson y Wodak, 2013). En este sentido, la prensa española potencia una imagen positiva de España frente a Italia, como se puede ver en el párrafo que sigue, en el que abiertamente se da la enhorabuena a España por su actitud solidaria y comprometida; nos encontramos ante un discurso que elogió la solidaridad española:

«Por entonces, Ximo Puig y Mónica Oltra, indignados por la indiferencia y el rechazo del Gobierno, proponían poner un barco a disposición de los refugiados para salvarlos del mar. Hoy, merced a Pedro Sánchez — que no olvida sus promesas—, Valencia no solo acoge, sino que cuenta con el apoyo del nuevo Gobierno. Enhorabuena para ambos, enhorabuena para España y enhorabuena para los millares de militantes anónimos que desde hace años luchan con firmeza y sin descanso para que la mano salvadora ciegue la odiosa mirada infligida a inmigrantes y refugiados» (El País, 18-06-2018).

Por un lado, Valencia se presenta como una ciudad solidaria, que está dispuesta a ayudar a personas en una situación de extrema necesidad. Por otro lado, España se presenta como un país que pretende ofrecer a la UE otra manera de abordar la inmigración:

«El alcalde de Valencia, Joan Ribó, previa consulta con el president Ximo Puig y la vicepresidenta Mónica Oltra, ofreció la ciudad hace una semana. El presidente español, Pedro Sánchez, no tuvo dudas. Era la oportunidad de ayudar a un grupo de náufragos 
APROXIMACIÓN AL DISCURSO DE LA ACOGIDA Y LA SOLIDARIDAD EN TORNO

AL RESCATE DEL BUQUE AQUARIUS EN UNA MUESTRA DE LA PRENSA ESPAÑOLA

y de demostrar a Europa que hay otra manera de abordar la inmigración. El gesto, la iniciativa, ha provocado un fuerte debate mediático, primer paso para que alcance a los políticos. La UE, en breve, celebrará un Consejo Europeo. Valencia será tema central de conversación, la Valencia solidaria que acogió al Aquarius» (La Vanguardia, 17-06-2018).

Se observa que son varios los ejemplos en los que se hace mención a la solidaridad española contrastandola con el rechazo de Italia y Malta, hecho que contribuye a potenciar el binomio "nosotros-ellos", España-Italia, y a destacar la buena acción de acoger a los inmigrantes en Valencia:

"Uno de nuestros principales rasgos de Valencia es la solidaridad y así lo hemos hecho saber al mundo durante estos días", ha añadido el alcalde de la capital, Joan Ribó, uno de los primeros en ofrecer la capital para acoger al Aquarius después del rechazo de Italia y Malta. Ribó, al igual que todos los que han tomado la palabra, ha agradecido el esfuerzo de todos los voluntarios y de las organizaciones participantes» (El País, 19-06-2018).

Presentar a España como un país que acoge y vela por el respeto a la vida y por los derechos de personas que vienen de países donde hay conflictos y violaciones de los DDHH, potencia un discurso de la acogida. Hablar de acoger sin matices es significativo y novedoso pues en general la tendencia del grupo mayoritario es a establecer diferencias con las personas que llegan (Richardson y Colombo, 2014). El elogio hacia la sociedad que acoge es evidente en las noticias relacionadas con el rescate del Aquarius, como se puede observar claramente en este ejemplo:

"La sociedad valenciana y otros territorios del Estado merecen toda nuestra admiración y respeto por la contundente respuesta que han dado ante la llegada de 629 personas a nuestros pueblos y ciudades. Una respuesta firme que clama querer acoger. Acoger sin matices. Para nuestro pueblo, acoger significa acoger. Significa poder dar garantías en el mantenimiento de la vida. Y significa atender a las necesidades de quienes vienen huyendo del hambre, las injusticias o los conflictos... Personas que están llegando y queremos entre nosotras. Como vecinos. Como vecinas. Las queremos entre nosotras y las queremos con derechos» (El Mundo, 17-06-2018).

Se vincula la autorrepresentación positiva con la solidaridad que se muestra en Valencia al acoger al Aquarius, tal y como se puede observar en el siguiente testimonio del presidente de la Generalitat Valenciana, Ximo Puig: "La reputación de la Comunitat Valenciana ha aumentado extraordinariamente no sólo en España, sino en toda Europa, simplemente por hacer aquello que nos corresponde" (La Vanguardia, 12-06-2018).

La siguiente afirmación de Mónica Oltra señala que esta acción va a servir para calificar a Valencia como ciudad acogedora, hecho que contribuirá a mejorar su reputación a nivel internacional: "Los ciudadanos se están volcando y que se va a poner a Valencia en el mapa como ciudad acogedora" (El País, 12-06-2018).

La solidaridad española no solo se destaca por medio de los testimonios de las personas con cargos políticos en España, sino que también la señalan personas con cargos en organismos internacionales, como es el caso del portavoz del Alto Comisionado de la Organización de Naciones Unidas (ONU) para los refugiados, Charlie 
Yaxley: "La oferta de la Moncloa es una gran muestra de solidaridad de España con los refugiados" (La Vanguardia, 11-06-2018). De manera similar, el portavoz de la Organización Internacional de las Migraciones (OIM) Leonard Doyle, vincula el gesto de España con ejercer un liderazgo que no es habitual: "La decisión del Gobierno español "demuestra liderazgo moral y legal, que es algo que escasea estos días en el mundo" (La Vanguardia, 11-06-2018).

La humanidad con la que se acoge a los inmigrantes en España contrasta con la falta de la misma manifestada por el Ministro del Interior italiano, al impedir la llegada del Aquarius a Italia. El siguiente párrafo de una noticia de El País, califica claramente a Italia y España de malo y bueno respectivamente:

«En el caso del Aquarius hubo elementos para armar una trama y, por eso, el sufrimiento de los náufragos se hizo visible. Existía un malote (Salvini), un bueno (el Gobierno de Sánchez), el coro de indiferentes (Malta y el resto de Europa), la coreografía de acogida (2.300 personas, entre voluntarios, médicos, psicólogos...) y los guionistas. En las costas andaluzas, en cambio, no hubo semejante despliegue» (El País, 22-06-2018).

Al hablar de dos grupos, en este caso Italia y España, con dos reacciones contrarias ante el mismo hecho, es necesario observar las estrategias que utiliza la prensa para representar a España de forma positiva, de modo que se la diferencia de Italia. Los ejemplos que siguen, contrastan la talla moral de España con la amenaza que supone el ministro italiano Salvini para los valores europeístas y democráticos:

"Matteo Salvini se ha convertido en la vergüenza de Europa, como Donald Trump lo es de una América que se forjó gracias a la acogida y flujos de extranjeros entre los que se incluye su esposa. El ministro del Interior italiano negó el desembarco del Aquarius, que el nuevo Gobierno español tuvo la talla moral de acoger, y niega ahora el del barco de la ONG Lifeline» (El País, 22-06-2018).

Los ejemplos que siguen sirven para contraargumentar a partir de la falta de solidaridad y acogida en Italia. La reacción del gobierno italiano va acompañada de un discurso nacionalista que justifica el rechazo a los inmigrantes y el cierre de los puertos italianos a los mismos, tal y como señalan Richardson y Colombo (2014, p. 537): "Discourse on national identity and insecurity jointly contributes to the justification and continuation of a variety of prejudicial positions vis-á-vis migrants and minority communities-rejection, avoidance, discrimination and expulsión". Así, en el siguiente ejemplo se observa que Salvini se refiere a los inmigrantes como prófugos (en contraste con la ciudadanía con derechos), y a su llegada a Italia como ser el felpudo de Europa. Nos encontramos, por tanto, ante un discurso xenófobo que rechaza a los inmigrantes y los considera inferiores; el rechazo de Salvini a acoger es claro y muestra claramente un 'nosotros' en una posición de poder que permite rechazar la entrada de 'ellos' y legitimar su decisión de no acoger al Aquarius:

"Están desembarcando en estos momentos en España 629 presuntos prófugos. Gracias al gobierno español. Espero que acoja otros 66.000. Nosotros no nos vamos a ofender". Así, con cierta provocación, el vicepresidente del gobierno y ministro del interior, Matteo Salvini, líder de la Liga, de extrema derecha, reaccionaba a la llegada de los 629 inmigrantes rescatados por la nave Aquarius a la que se cerraron los puertos italianos. 
APROXIMACIÓN AL DISCURSO DE LA ACOGIDA Y LA SOLIDARIDAD EN TORNO

AL RESCATE DEL BUQUE AQUARIUS EN UNA MUESTRA DE LA PRENSA ESPAÑOLA

El caso ha sido utilizado como un gran spot propagandístico por Salvini, quien evoca constantemente el caso Aquarius, como ayer hizo en un mitin electoral en Seregno, en la provincia de Monza y Brianza, al norte de Italia, y después en Twitter: "La Aquarius llega a España. Por primera vez una nave que parte desde Libia con destino a Italia atraca en un país diverso. Es una señal de que algo está cambiando, no somos ya el felpudo de Europa» (ABC, 18-06-2018).

Sin embargo, el discurso que se asocia con España, en concreto con Valencia, es el de la solidaridad. En el ejemplo que sigue, el binomio "nosotros-ellos" se traduce en "nosotros" que rescatamos, ayudamos y compartimos nuestros recursos mientras que Italia rechaza y se niega a ofrecer cualquier tipo de ayuda: "La ciudad ha recibido con los brazos abiertos a las 630 personas rescatadas la semana pasada por el buque Aquarius y que después fueron rechazadas por el gobierno italiano" (La Vanguardia, 18-06-2018).

Además, el posicionamiento de España ante la crisis humanitaria en que se encuentra el buque Aquarius se considera como una respuesta alternativa a la política europea relacionada con la inmigración; se contrasta el gesto comprometido de España con la indiferencia general, hecho que contribuye no solo a la representación positiva del país, sino también a representarlo como un modelo a seguir:

«El gesto del Gobierno español no es la solución, es sólo una llamada a la decencia europea frente al auge de la indiferencia generalizada. Debe traducirse en una propuesta de acción que, desde España, retome la política común europea en materia de inmigración de modo ofensivo y no sólo defensivo como se ha hecho hasta ahora" (El País, 18-06-2018).

De igual forma, la decisión de España se destaca por ser el único país de Europa que se ofrece a acoger ante esta emergencia. Calificar de inhumana e impresentable el comportamiento de Malta e Italia cerrando sus puertos contribuye a elogiar la actitud solidaria de España y a potenciar las diferencias entre unos países y otros. En este ejemplo, se ensalza la España de Pedro Sánchez, de modo que se quiere marcar una orientación ideológica respecto a Italia y Malta y quizás respecto al gobierno anterior:

"Tras ocho días en altamar, los tres barcos que han acogido a los 630 inmigrantes del Aquarius llegaron ayer al puerto de Valencia, después de que el Gobierno de Pedro Sánchez haya sido el único en toda Europa que ofreció una solución inmediata a la inhumana e impresentable conducta mantenida por los Ejecutivos de Italia o Malta. Había una prioridad humanitaria, que era rescatar a esas 630 personas, 126 de ellos niños desprotegidos y solos, y el país ha cumplido con creces. El gesto es sin duda hermoso humanamente y pone en valor la solidaridad del pueblo español» ( $A B C$, 18-06-2018).

El comisario de Inmigración de la Comisión Europea Dimitris Avramopoulos considera la decisión de España como un caso de solidaridad real pues España muestra un compromiso con una situación de emergencia y da un ejemplo al resto de países de la UE: "(al hablar del Gobierno español) La solidaridad real puesta en práctica, tanto hacia las personas vulnerables como hacia el resto de los miembros de la UE". ( $L a$ Vanguardia, 11-06-2018). 
APROXIMACIÓN AL DISCURSO DE LA ACOGIDA Y LA SOLIDARIDAD EN TORNO

AL RESCATE DEL BUQUE AQUARIUS EN UNA MUESTRA DE LA PRENSA ESPAÑOLA

La decisión del gobierno español de ofrecer España para la llegada de inmigrantes con el fin de evitar una crisis humanitaria es vista con buenos ojos tanto por los organismos internacionales como por las ONGs. Dicha decisión posiciona a España ante la UE como defensora de políticas migratorias que defienden los DDHH y que respete las políticas internacionales:

"Desde "liderazgo moral" a "solidaridad real", las alabanzas de las instituciones europeas y de organismos y oenegés internacionales al Gobierno español se han repetido este lunes por la tarde después de que el Ejecutivo central haya hecho público a través de un comunicado que ofrece València como "puerto seguro" para el buque Aquarius, en lo que ha sido definido como un gesto personal del presidente del Gobierno, Pedro Sánchez» (La Vanguardia, 11-06-2018).

La representación de Italia como país frío que rechaza a los inmigrantes y que establece un contraste con la solidaridad española se refuerza aún más al señalar que Francia está dispuesta a colaborar con España en la acogida de los inmigrantes, cumpliendo así con el derecho internacional. En este sentido, "nosotros" en el contexto que nos ocupa también puede entenderse como España y Francia, pues el Gobierno de Emmanuel Macron muestra su apoyo al gobierno de Sánchez en todo momento dando la posibilidad de que los inmigrantes puedan presentar solicitud de asilo en Francia. La cooperación entre España y el país vecino señala que hay distintos modos de abordar la inmigración en la UE:

"La vicepresidenta del Gobierno, Carmen Calvo, ha afirmado este lunes que casi la mitad de los migrantes llegados a España en el operativo del buque Aquarius quieren ir a Francia, país que de momento parece que va a colaborar. Lo ha dicho Calvo a su llegada a la sede socialista madrileña de Ferraz, donde ha calificado de "situación bastante idónea" la oferta de Francia pues "se trata de cooperar y de cumplir la normativa europea y el derecho internacional» (La Vanguardia, 18-06-2018).

El discurso solidario no solo está en manos de los/as políticos/as, sino que también se encuentra en personas que pertenecen a las ONGs implicadas en el rescate del Aquarius. Así, David Beversluis, uno de los médicos a bordo de la embarcación de Médicos Sin Fronteras (MSF), también critica la postura del gobierno italiano y la contrasta con la solidaridad que han mostrado las ONGs que han tomado parte activa en el rescate de las/os inmigrantes:

"Todo el equipo de MSF y SOS Méditerranée a bordo del Aquarius nos solidarizamos con estas 630 personas vulnerables y defenderemos a todas y cada una de ellas; se han jugado la vida para dejar atrás horribles condiciones de esclavitud en Libia y se han quedado tiradas en el mar. En vez de abrir los brazos para acoger a quienes han pasado por este sufrimiento, a estos hombres, mujeres y niños, el Gobierno italiano les ha dado la espalda y ha vuelto a poner sus vidas en riesgo con gran temeridad» ( $E l$ Mundo, 18-06-2018).

El hecho de que la Vicepresidenta del Gobierno, Carmen Calvo, considere magnífica la gestión de España en el rescate del Aquarius y se vincule esta iniciativa solidaria en una situación de emergencia con la imagen que España ha de tener a nivel 
internacional sugiere que el ofrecimiento a estos inmigrantes se relaciona con la oportunidad de mejorar la imagen de España:

"Calvo ha resaltado que la gestión española ha sido "magnífica", tanto por parte de las ONG que colaboran como por parte de las autoridades autonómicas y los ministerios implicados. Se ha dado "una imagen muy solvente de nuestro país y nos devuelve al crédito para el que todos debemos contribuir" con el fin de que esta "sea la imagen de España y no otras", ha recalcado» (La Vanguardia, 18-06-2018).

La cuestión de mejorar la imagen de España parece vincularse con los casos de corrupción política que se han detectado en España en general y en Valencia en particular. Esto contrasta con considerar ahora la ciudad como modelo de comportamiento solidario, respetuoso y acogedor:

"Valencia, asociada en exceso en el pasado a la corrupción y al saqueo de las instituciones, se ha convertido hoy en paradigma de la solidaridad, de la humanidad, de la protección de los derechos humanos" (La Vanguardia, 17-06-2018).

Aunque el discurso que elogia a España está en manos de políticos, una vez que el rescate se ha realizado, también encontramos algunos testimonios de personas con cargos en ONGs, el presidente de Cruz Roja en Valencia, Rafael Gandía, se refiere con orgullo a Valencia y a su voluntariado: "Valencia ha sido capital mundial de la solidaridad". "Estamos orgullosos de nuestro voluntariado", ha enfatizado (El Mundo, 18-06-2018).

\section{Discusión}

La decisión del recién elegido gobierno socialista de acoger al buque Aquarius en el puerto de Valencia es relevante porque en torno a esta decisión surge un discurso relacionado con la acogida y la solidaridad debido a una cuestión humanitaria. El discurso de la prensa muestra la decisión del gobierno de Sánchez de forma claramente positiva pues esta lleva consigo proyectar una imagen positiva de España no solo a nivel europeo sino también a nivel mundial. Además, se presenta a la sociedad española en general y a la valenciana en particular como solidaria de modo que se da una imagen positiva del grupo mayoritario, hecho que contribuye a la creación de opinión pública. Llama la atención que dicho discurso generado en torno a la llegada del Aquarius contrasta con el discurso que suele predominar en la prensa relacionado con la inmigración que se caracteriza por presentar a los inmigrantes como personas-problema, aspecto que contribuye a desarrollar patrones de discriminación en la sociedad española. Generar discursos positivos y solidarios en torno a la llegada del Aquarius tiene importancia, especialmente en estos años en los que se ha de trabajar por conseguir los Objetivos de Desarrollo Sostenible (ODS) propuestos para el año 2030. En concreto, esta investigación contribuye a promover el objetivo 16, al que nos hemos referido anteriormente.

El discurso de la acogida y la solidaridad analizado en este artículo debería ser el primer paso para llevar a cabo acciones solidarias que eliminaran las actitudes racistas o de rechazo hacia las personas refugiadas. En este sentido, potenciar discursos 
positivos que promuevan la integración y la solidaridad contribuye a avanzar en que la justicia social sea real en nuestras sociedades, de modo que los países sean más equitativos, justos y solidarios (Hagen-Zanker, Mosler Vidal y Sturge, 2017). Sin embargo, al comparar los resultados de este estudio con otros similares (Martínez Lirola, 2020, en prensa; Solves Almela y Arcos Urrutia, 2020), se observa que al hacer un seguimiento posterior de lo que ocurre con el caso y su tratamiento mediático, desaparece el discurso solidario que ha sido ilustrado en este artículo y que se caracteriza por elogiar la actitud solidaria de la población española por medio del compromiso con la acogida de los inmigrantes en una situación de emergencia. Otra característica de este discurso consiste en optar por la autorrepresentación positiva de España, que va unida al contraste con la falta de solidaridad que se observa en Italia.

Las referencias al Aquarius y las noticias relacionadas con el tema tras el año 2018 son casi inexistentes, pues solo se hace referencia a la situación de los inmigrantes que llegaron cuando se cumplen el año o los dos años de la llegada y en las pocas de las noticias que se publican se hace mención a que muchos de los inmigrantes que llegaron siguen sin regularizar su situación. Es decir, este discurso de la solidaridad desapareció poco después, tanto por parte del Gobierno de España como por parte de la mayoría de la sociedad civil.

Hemos de señalar que en las noticias analizadas las personas del grupo mayoritario aparecen llamadas por su nombre y el cargo que ocupan, tanto en la política como en las ONGs. Esto contrasta con las referencias a los inmigrantes a los que se menciona de forma general, sin especificar nada sobre ellos, como individuos anónimos (van Leeuwen, 2008, p. 39), es decir, generalmente, no se dice nada sobre su identidad, las razones por las que vienen y sus situaciones personales en sus países de origen. Las referencias a los inmigrantes los presentan como víctimas pasivas: al no haber triunfado en su proyecto migratorio, necesitan ser rescatados y protegidos por la población española, que asume un papel activo. Así, se potencia un imaginario icónico de los inmigrantes que no favorece la inclusión (Martín Nieto, 2009).

Tras el análisis presentado en este artículo, nos seguimos haciendo estas preguntas: ¿ha contribuido el discurso solidario y humanitario que surgió en 2018 en torno al Aquarius a cambiar algunas actitudes racistas en la población española? ¿Va a contribuir a hacer políticas europeas humanas y solidarias en lo que respecta a inmigración? Esperemos que así sea en el futuro, aunque no se haya hecho por el momento. En este sentido, conviene precisar que lo fugaz de este discurso de la solidaridad no ha servido para garantizar que los inmigrantes hayan estado en el centro del discurso periodístico, haciendo una reivindicación de la defensa de los DDHH y de la humanidad.

\section{Conclusiones}

La decisión política de acoger al Aquarius lleva consigo acciones ejecutadas por las ONGs y las fuerzas de seguridad del Estado. Esto es narrado por la prensa de tal modo que se genera un discurso de la acogida y la solidaridad. Así, se deja de lado el discurso tradicional que fomenta el rechazo a los inmigrantes que llegan a España. Ofrecer un análisis de dicho discurso sirve para ofrecer una aproximación a sus características y a visibilizar a las personas que lo pronuncian, a la vez que se profundiza en las relaciones entre la lengua y la realidad migratoria. 
APROXIMACIÓN AL DISCURSO DE LA ACOGIDA Y LA SOLIDARIDAD EN TORNO

AL RESCATE DEL BUQUE AQUARIUS EN UNA MUESTRA DE LA PRENSA ESPAÑOLA

El binomio "nosotros-ellos" contrasta la actitud acogedora de España con el rechazo de Italia. Además, se potencian las diferencias entre "nosotros", la sociedad española que acoge (activa) y "ellos", los inmigrantes que son rescatados y necesitan ayuda y recursos (pasivos). Por tanto, no se sitúa a los dos grupos de la población al mismo nivel por dos razones fundamentales:1) por ser la población española la que ayuda y los inmigrantes los que la reciben; 2) por el escaso número de testimonios de inmigrantes comparado con el número de las personas con cargos políticos, y en menor medida con la de miembros de ONGs.

El análisis presentado en este artículo muestra que, aunque el rescate del buque Aquarius lleva consigo la creación de un discurso que elogia la acogida y la solidaridad de España en contraste con Italia; también se observan algunos ejemplos que invitan al rechazo al hacer referencia al efecto llamada o a la llegada masiva. La perspectiva del ACD permite observar cómo la lengua se relaciona con el poder del grupo mayoritario para presentarse de forma positiva y para narrar el rescate del buque Aquarius con un discurso humanitario que favorece la acogida de los inmigrantes rescatados.

\section{Bibliografía}

$A B C, 10$ junio-10 julio, 2018. http://www.abc.es

ACNUR (Alto Comisionado de las Naciones Unidas para los Refugiados). 2016. Informe anual 2016. Madrid: ACNUR.

ACNUR (Alto Comisionado de las Naciones Unidas para los Refugiados). 2017. Informe anual 2017. Madrid: ACNUR.

Aguilar-Idáñez, M.J. y Buraschi, D. (2017). Migrantes y refugiados: apuntes clave para un nuevo relato. Revista Lusófona de Educação, 37, pp. 103-116. doi: 10.24140/issn.1645-7250.rle37.07

Agustín García, O. y Bak Jørgensen, M. (2016). Against pessimism: A time and space for solidarity. En O. García Agustín y M. Bak Jørgensen (Eds.), Solidarity without borders: Gramscian perspectives on Migration and Civil Society Alliances, pp. 223-233. Londres: Pluto Press.

Agustín García, O. y Bak Jørgensen, M. (eds.) (2018). Solidarity and the 'Refugee Crisis' in Europe. Cham: Palgrave Macmillan.

Amnistía Internacional (2015). Miedo y vallas. Los planteamientos de Europa para contener a las personas refugiadas. Londres. Amnistía Internacional.

Anderson, B. (2013). Us and them? The dangerous politics of immigration control. Oxford: Oxford University Press.

Arango, J. (2002). La fisonomía de la inmigración en España. El campo de las ciencias y las artes, 139 ("El nuevo orden demográfico"). Madrid: Servicio de Estudios BBVA.

Arenas-Hidalgo, N. (2016). Flujos masivos de población y seguridad. La crisis de personas refugiadas en el Mediterráneo. Araucaria. Revista Iberoamericana de Filosofía, Política y Humanidades, 18(36), pp. 339-372.

Bañón, A. (2019). Medios de comunicación y procesos migratorios: reflexiones para el debate. En Comité Especializado de Inmigración (Eds.), El fenómeno migratorio en España: reflexiones desde el ámbito de la Seguridad Nacional, pp. 353-359. Madrid: Ministerio de la Presidencia, Relaciones con las Cortes e Igualdad.

Bañón, A. M. (2002). Discurso e inmigración. Propuesta para el análisis de un debate social. Murcia: Universidad de Murcia.

Bañón Hernández, A. (2007). Discursos periodísticos y procesos migratorios. San Sebastián: Tercera Prensa.

Bañón Hernández, A. M. y Romero, S. R. (2013). Ánimo. Estamos con vosotros: Messages of solidarity written in a visitors' book during a sit-in conducted by a group of immigrants in Spain. Discourse and Society 24(1), pp. 3-26. 
APROXIMACIÓN AL DISCURSO DE LA ACOGIDA Y LA SOLIDARIDAD EN TORNO

AL RESCATE DEL BUQUE AQUARIUS EN UNA MUESTRA DE LA PRENSA ESPAÑOLA

Bednarek, M. y Caple, H. (2017). The discourse of news values. How news organizations create newsworthiness. Oxford: Oxford University Press.

Caicedo, D. A. (2016). Alien, illegal, and undocumented: labeling, context, and worldview in the immigration debate and in the lives of undocumented youth. Nueva York, NY: CUNY Academic Works.

Casero, A. (2007). Discurso mediático, inmigración e ilegalidad: legitimar la exclusión a través de la noticia. En R. Zapata Barrero y T. Van Dijk (Eds.), Discursos sobre la inmigración en España. Los medios de comunicación, los parlamentos y las administraciones, pp. 69-90. Barcelona: Fundación CIDOB.

Cruz Moya, O. (2005). Las migraciones en el discurso periodístico almeriense del siglo XX. Análisis crítico de tres corpus de noticias. Tesis doctoral. Sevilla: Universidad Pablo de Olavide.

Cruz Moya, O. (2020). De "lobos solitarios" a "carpas africanas": estrategias de despersonalización en las metáforas empleadas por el discurso periodístico en torno a los refugiados. Tonos Digital, 38(1), pp. 1-15.

El Mundo, 10 junio-10 julio, 2018. http://www.elmundo.es

El País, 10 junio-10 julio, 2018. http://www.elpais.es

Fairclough, N. (1995). Critical discourse analysis. The critical study of language. Londres: Longman.

Faure, R., Gavas, M. y Knoll, A. (2015). Challenges to a comprehensive EU migration and asylum policy. Londres y Maastricht: ECDP/ODI.

Hagen-Zanker, J., Mosler Vidal, E. y Sturge, G. (2017). Social protection, migration and the 2030 Agenda for sustainable development. Londres: ODI.

KhosraviNik, M. (2009). The representation of refugees, asylum seekers and immigrants in British newspapers during the Balkan conflict (1999) and the British general election (2005). Discourse E Society, 20(4), pp. 477-498.

KhosraviNik, M. (2010). Actor descriptions, action attributions, and argumentation: towards a systematization of CDA analytical categories in the representation of social groups. Critical Discourse Studies, 7(1), pp. 55-72.

KhosraviNik, M. (2014). Immigration discourses and critical discourse analysis. En C. Hart y P. Cap (Eds.), Contemporary critical discourse studies, pp. 501-519. Bloomsbury Publishing.

Konstantinidou, C. y Michailidou, M. (2014). Foucaldian discourse analysis: Photography and the social construction of immigration in the Greek national press. En D. Machin (Ed.), Visual Communication, pp. 99-133. Berlín: Mouton de Gruyter.

La Vanguardia, 10 junio-10 julio, 2018. http://www.lavanguardia.com

Martín Nieto, R. (2009). El Tercer Mundo representado: la imagen como nido de estereotipos. ICONO, 14(5), pp. 1-19.

Martínez Lirola, M. (2016). Multimodal representation of Sub-Saharan immigrants as illegal: deconstructing their portrayal as victims, heroes and threats in a sample from the Spanish press. Bulletin of Hispanic Studies, 93(4), pp. 343-360.

Martínez Lirola, M. (2017a). Linguistic and visual strategies for portraying immigrants as people deprived of human rights. Social Semiotics, 27(1), pp. 21-38. doi: $10.1080 / 10350330.2015 .1137164$

Martínez Lirola, M. (2017b). Discursive legitimation of criminalization and victimization of sub-Saharan immigrants in Spanish El País and $A B C$ newspapers". En J. Chovanec y K. Molek-Kozakowska (Eds.), Representing the other in European media discourses, pp. 136-154. Amsterdam/Philadelpia: John Benjamins. doi: 10.1075/dapsac.74.07mar

Martínez Lirola, M. (2020). Explorando el discurso de la prensa en torno a la defensa de los derechos humanos en el rescate del buque Aquarius. Migraciones, 49, pp. 147-170. doi: 10.14422/mig.i49.y2020.006

Martínez Lirola, M. (en prensa). Critical analysis of the main discourses of the Spanish press about the rescue of the ship Aquarius. Communication \& Society. 
APROXIMACIÓN AL DISCURSO DE LA ACOGIDA Y LA SOLIDARIDAD EN TORNO

AL RESCATE DEL BUQUE AQUARIUS EN UNA MUESTRA DE LA PRENSA ESPAÑOLA

Milner, M. W. (2012). The discursive construction of global poverty: Social justice in media discourse. En K.V. Korostelina (Ed.), Forming a Culture of Peace. Reframing Narratives of Intergroup Relations, Equity, and Justice, pp. 131-164. Nueva York: Palgrave Macmillan.

Moore K, Gross B. y Threadgold T. (eds.). (2012). Migrations and the Media. Nueva York: Peter Lang.

Naciones Unidas, Asamblea General, 2015. Objetivos de Desarrollo Sostenible. A/69/L.85. 12 agosto 2015. Disponible en: http://www.un.org/sustainabledevelopment/es/

Nelson, R.L. y Davis-Wiley, P. (2018). Illegal or undocumented: An analysis of immigrant terminology in contemporary American media. International Journal of Social Science Studies, 6(6), pp. 8-15.

Niñoles Galvañ, A. y Ortega-Giménez, C. (2020). Discurso del odio en radio: análisis de los editoriales de las cadenas COPE y SER tras la llegada del Aquarius a España. Miguel Hernández Communication Journal, 11(1), pp. 117-138. doi: 10.21134/mhcj.v11i0.317

Osorio Ramírez, A. (2012). Migraciones internacionales: nuevos desafíos para la cohesión social. El caso de los ex yugoslavos en Quebec. Boletín de Antropología, 26(43), pp. 86-104.

Pries, L. (2018). Entre la bienvenida y el rechazo: la "Crisis de los refugiados" en Europa. POLIS, 14(2), pp. 71-96.

Reina-Vanegas, F. C. (2016). \#Refugiados. Discursos de alteridad de población solidaria en Facebook. Revista Eleuthera, 14, pp. 87-104. doi: 10.17151/eleu.2016.14.6.

Richardson, J. E. y Colombo, M. (2014). Race and Immigration in Far-and Extreme-Right European Political Leaflets. En C. Hart y P. Cap (Eds.), Contemporary critical discourse studies, pp. 521-542. Londres: Bloomsbury Publishing.

Richardson, J. y Wodak, R. (2013) The Impact of Visual Racism: Visual Arguments in Political Leaflets of Austrian and British Far-Right Parties. En R. Wodak, (Ed.), Critical discourse analysis. Vol. IV. Applications, interdisciplinary perspectives and new trends, pp. 245-274. Londres: Sage.

Romano, M. (2019). 'Refugees are streaming into Europe': An image-schema analysis of the Syrian Refugee crisis in the Spanish and British press. Complutense Journal of English Studies, 27, pp. 39-57.

Solves Almela, J. y Arcos Urrutia, J. M. (2020). El Aquarius desembarca en la prensa española. Tonos Digital, 38(1), pp. 1-27.

van Dijk, T. A. (2005a). Racism and discourse in Spain and Latin America. Amsterdam/Philadelphia: John Benjamins.

van Dijk, T. A. (2005b). Discurso de las élites y racismo institucional. En M. Lario Bastida (Ed.), Medios de comunicación e inmigración, pp. 16-34. Murcia: Convivir sin Racismo: CAM Obra Social.

Van Leeuwen, T. (2008). Discourse andpPractice. New tools for critical discourse analysis. Oxford: Oxford University Press.

Wodak, R. (2011). Critical linguistics and critical discourse analysis. En J. Zienkowski, J-O Östman, y J. Verschueren (Eds.), Discursive pragmatics, pp. 50-70. Amterdam/Philadelphia: John Benjamins.

Wodak, R. y Meyer, M. (2009). Critical discourse analysis: History, agenda, theory and methodology. En R. Wodak y M. Meyer (Eds.), Methods of critical discourse analysis, pp. 1-33. 2 ed. Londres: Sage.

Wodak, R. y Reisigl, M. (2001). Discourse and racism. En D. Tannen, D. Schiffrin y H. Hamilton (Eds.). The handbook of discourse analysis, pp. 372-397. Oxford: Blackwell. 